\title{
LA MOTIVACIÓN Y EL COMPROMISO ORGANIZACIONAL EN LOS DOCENTES DE LOS PROGRAMAS ESPECIALES DESCENTRALIZADOS DE LA UNIVERSIDAD NACIONAL DE PIURA, 2017
}

THE MOTIVATION AND ORGANIZATIONAL COMMITMENT IN TEACHERS OF THE DECENTRALIZED SPECIAL PROGRAMS OF THE NATIONAL UNIVERSITY OF PIURA, 2017

NOE ALEXANDER NÚÑEZ BARRIA MARIO ENRIQUE RUESTA YARLEQUE EDDY WILLIAM GIVES MUJICA ERNESTO QUEZADA POICON LUIS RAMON TRELLES POZO 



\title{
LA MOTIVACIÓN Y EL COMPROMISO ORGANIZACIONAL EN LOS DOCENTES DE LOS PROGRAMAS ESPECIALES DESCENTRALIZADOS DE LA UNIVERSIDAD NACIONAL DE PIURA, 2017
}

\author{
THE MOTIVATION AND ORGANIZATIONAL \\ COMMITMENT IN TEACHERS OF THE \\ DECENTRALIZED SPECIAL PROGRAMS OF THE \\ NATIONAL UNIVERSITY OF PIURA, 2017
}

\author{
Noe Alexander Núñez Barria \\ Mario Enrique Ruesta Yarleque \\ Eddy William Gives Mujica \\ Ernesto Quezada Poicon \\ Luis Ramon Trelles Pozo
}

Palabras clave: motivación, compromiso, docente, proedunps, UNP

Keywords: motivation, commitment, teaching, proedunps, UNP

\section{RESUMEN}

La presente investigación es un la relación existente entre la motivación estudio de tipo descriptivo - correlacio- y el compromiso organizacional de los nal que tiene como objetivo identificar docentes de los Programas Especiales 
Descentralizados - PROEDUNP's de la Universidad Nacional de Piura, en el año 2017. Para ello se utilizaron los instrumentos de investigación TCM y R-MAWS que fueron administrados a 168 docentes en cinco de los programas especiales descentralizados de la Universidad Nacional de Piura (Paita, Sechura, Talara, Huancabamba y Chulucanas) con los cuales se llegó a la conclusión de que la motivación se encuentra en niveles favorables principalmente en las siguientes dimensiones: motivación intrínseca, regulación identificada y regulación introyectada. En lo que corresponde a la regulación externa, existe una valoración media de esta dimensión; con respecto al compromiso organizacional, y en relación a la convivencia dentro del entorno de trabajo, circunstancias de cercanía e identificación con la institución, así como de la predisposición de desarrollar sus actividades, el $100 \%$ de los encues- tados demuestran gratitud para con la institución. Asimismo, se debe señalar que el nivel de compromiso organizacional es favorable principalmente en la dimensión normativa donde alcanza valores altos; en lo que respecta a las posibles alternativas de continuidad y al temor de dejar la institución, los valores encontrados son medios. Debemos establecer que el deseo de pertenecer a la organización y de seguir en ella también presenta niveles favorables para la institución.

Como conclusión final se establece que la relación existente entre la motivación y el compromiso organizacional de los docentes de los Programas Especiales Descentralizados - PROEDUNP's de la Universidad Nacional de Piura, en el año 2017, según el coeficiente de correlación de Spearman, es de 0,638; lo que indica que existe una relación positiva entre las variables.

\section{ABSTRACT}

The present research is adescriptive - correlational type study. The objective is to establish the existing relationship between the motivation and organizational commitment of the teachers of the Decentralized Special Programs - PROEDUNP of the National University of Piura, in the year 2017. "For this, the research instruments (TCM and R-MAWS) were used on 168 teachers in five of the decentralized special programs of the National University of Piura (Paita, Sechura, Talara, Huancabamba and Chulucanas), arriving at the conclusion that the motivation is at favorable levels mainly in the following dimen- 
sions: motivation intrinsic, identified regulation and introjected regulation, in what corresponds to external regulation there is an average assessment of this dimension, with respect to organizational commitment and in relation to coexistence within the work environment, the circumstances of closeness and identification with the institution, as well as the willingness to develop its $s$ activities, $100 \%$ of respondents show their gratitude to the institution. It should be noted that the level of organizational commitment is favorable mainly in the normative dimension when high values are reached, as regards the possible alternatives of continuity and in regard to the fear of leaving the institution the values found by means. A tool for the development of the family and the organizational development of the teachers of the Decentralized Special Programs PROEDUNP 's of the National University of Piura, in the year 2017, according to the Spearman correlation coefficient is of 0.638; Which indicates that there is a positive relationship between the variables.

\section{INTRODUCCIÓN}

En la actualidad, las empresas brindan relevancia al capital humano, se considera que en una organización son múltiples las variables que a diario se reflejan en los trabajadores y que son de gran influencia para cada organización.

Al respecto, Ruíz (2013) manifiesta que uno de los aspectos más relevantes e importantes en las organizaciones es el compromiso organizacional, ya que este contribuye a la mejora, logro de objetivos y compromiso del trabajador con la organización. Asimismo, considera que el compromiso y la motivación laboral, son aspectos que suelen estar unidos y que al ser adecuados, contribuyen positivamente a toda la organización.
Ante esto, la presente investigación busca evaluar el compromiso organizacional y la motivación en los Docentes de los Programas Especiales Descentralizados de la UNP en el año 2017.

Se parte de la base de que es política de la actual Gestión de la Universidad Nacional de Piura, avizorar y poner en práctica la promoción, difusión, actuación de actividades académicas inclusivas en el ámbito regional, para que sus ciudadanos tengan la oportunidad de acceder al conocimiento científico, humanístico y tecnológico, en las diversas modalidades que brinda la Universidad: Preparación Preuniversitaria, Pregra- 
do, Facultades y Escuelas Profesionales, Posgrado, Centro de Idiomas y Escuela Tecnológica.

Este crecimiento de manera rápida ha presentado oportunidades de acceso a la educación orientada hacia la población de zonas lejanas, en este sentido también se enfatiza que se debe brindar una enseñanza de calidad acorde a las expectativas generadas.

Asimismo, para poder ofrecer servicio de calidad, el compromiso de los docentes que viajan a cada sede descentralizada debe ser el adecuado. Por ello, los docentes deben ser motivados dentro de lo establecido y permitido por la reglamentación vigente de la Universidad Nacional de Piura.

Esta investigación nace con el fin de establecer la relación de la motivación y el grado de compromiso de los docentes para con los Programas Especiales Descentralizados - PROEDUNP's.

Con este fin, el presente estudio responderá a la siguiente interrogante: ¿cuál es la relación entre la motivación y el compromiso organizacional de los docentes de los programas especiales descentralizados de la universidad nacional de Piura, en el año 2017?

Teniendo un alcance correlacional y basándonos en la literatura de Luthans (2008) se define al compromiso organizacional como la entrega y responsabilidad del trabajador con su empresa, para que desarrolle un trabajo óptimo además de las estrategias que la empresa realice para fomentar el compromiso promoviendo un espacio de trabajo estimulante. Katz \& Kahn (1977 citados por Zamora, 2009) fueron unos de los primeros que definieron el término; para ellos, el compromiso organizacional es la interiorización de los empleados con las metas de la institución, generando una coherencia entre estos y las metas personales. Y Gagné (2010) quien sostiene que la motivación puede definirse como una condición del organismo, cuya energía origina determinadas conductas, relacionadas con el ambiente para alcanzar un fin significativo.

\section{METODOLOGÍA}

\section{Procedimiento de la investigación}

Para el análisis descriptivo de las dimensiones de las variables estudiadas fue necesario establecer rangos bajo, medio y alto para Motivación y Compro- miso Organizacional; para ello se procedió a realizar el siguiente procedimiento:

A continuación, presentamos la valoración de cada una de las dimensiones de la variable Motivación, según el 
número de ítems agrupados en cada una de ellas y los tres rangos establecidos:

Tabla 1. Puntuación según dimensión - Motivación

\begin{tabular}{llll}
\hline & Bajo & Medio & Alto \\
\hline Desmotivación (3 ítems) & Hasta 9 & $10-16$ & 17 a más \\
\hline Regulación Externa (6 ítems) & Hasta 18 & $19-31$ & 32 a más \\
\hline Regulación Introyectada (4 ítems) & Hasta 12 & $13-21$ & 22 a más \\
\hline Regulación Identificada (3 ítems) & Hasta 9 & $10-16$ & 17 a más \\
\hline Motivación Intrínseca (3 ítems) & Hasta 9 & $10-16$ & 17 a más \\
\hline
\end{tabular}

Fuente: Elaboración Propia.

A continuación, presentamos la ganizacional, según el número de items valoración de cada una de las dimen- agrupados en cada una de ellas y los tres siones de la variable Compromiso Or- rangos establecidos:

Tabla 2. Puntuación según dimensión - Compromiso Organizacional

\begin{tabular}{lccc}
\hline & Bajo & Medio & Alto \\
\hline Afectiva (6 ítems) & Hasta 18 & $19-31$ & 32 a más \\
\hline Normativa (6 ítems) & Hasta 18 & $19-31$ & 32 a más \\
\hline Continuidad (6 items) & Hasta 18 & $19-31$ & 32 a más \\
\hline
\end{tabular}

Fuente: Elaboración Propia.

Finalmente, se correlacionaron las dimensiones de la variable Motivación y los valores generales de la variable Compromiso Organizacional mediante el coeficiente de correlación de Spearman.

\section{Unidad de estudio}

\section{Población}

La población estuvo conformada por el Personal docente de los PROEDUNP's, en un total de 347. 


\section{Muestra}

Para la determinación de la muestra se utilizó el Software Epidat 4.2 (Programa para Análisis Epidemiológico de Datos), asumiendo para ello un nivel de confianza del 95,0\%; una proporción esperada de $70,0 \%$; asimismo un error de precisión del 5,0\%, consideraciones que dieron como resultado que nuestra muestra analizada estuviera compuesta por 168 docentes de las sedes descentralizadas de la Universidad Nacional de Piura.

\section{Datos}

Tamaño de la Población:

Proporción Esperada:

Probabilidad de Error:

Nivel de Confianza: $30,0 \%$ $95,0 \%$

\section{Resultados}

Tabla 3. Determinación de la Muestra

\begin{tabular}{cc}
\hline Precisión (\%) & Tamaño de la Muestra \\
\hline 5,000 & 168 \\
\hline
\end{tabular}

Fuente: Epidat 4.2 (Programa para Análisis Epidemiológicos de Datos). Diseño: Propio.

Al tratarse de subgrupos que no tienen el mismo tamaño de estrato (subgrupo de poblaciòn, para el caso, las sedes descentralizadas), fue necesario un reparto proporcional al mismo de esos subgrupos que componen nuestra 347 muestra representativa, quedando esta$70,0 \%$ blecidos según se muestra en la tabla 4.

Tabla 4. Determinación de la Muestra Estratificada

\begin{tabular}{lcc}
\hline Estrato & Tamaño del Estrato & Tamaño de la Muestra \\
\hline Chulucanas & 106 & 51 \\
\hline Talara & 60 & 29 \\
\hline Sechura & 78 & 38 \\
\hline Paita & 73 & 35 \\
\hline Huancabamba & 30 & 15 \\
\hline Total & 347 & 168 \\
\hline
\end{tabular}

Fuente: Epidat 4.2 (Programa para Análisis Epidemiológicos de Datos).

Diseño: Propio. 


\section{INSTRUMENTOS}

\section{Métodos de Investigación}

En la presente investigación se empleó el método descriptivo, correlacional, que se complementó con los métodos de análisis deductivo e inductivo según se requirió.

\section{Técnicas de Investigación}

Se utilizó como técnica la en- cuesta y para evaluar los niveles de Compromiso Laboral se aplicó como instrumento de medición el Modelo de Tres Componentes del Compromiso (TCM, siglas en inglés) de Meyer y Allen (1997), que consta de 18 reactivos, 6 reactivos para cada una de las subescalas o dimensiones; con un alfa de 0,82 .

\begin{tabular}{l|l|c}
\hline \multicolumn{1}{c}{ Dimensión } & \multicolumn{1}{c}{ Indicadores } & \multicolumn{1}{c}{ Preguntas } \\
\hline \multirow{2}{*}{ Afectiva } & $\begin{array}{l}\text { Identificación e involucramiento de los trabajadores con la orga- } \\
\text { nización. Deseo de pertenecer a la organización (quieren/desean } \\
\text { hacerlo) }\end{array}$ & $6,9,12,14,15,18$ \\
\hdashline Normativa & $\begin{array}{l}\text { Lealtad, sentido de organización. Sentido de obligación hacia la } \\
\text { organización (deben están obligados) }\end{array}$ & $2,7,8,10,11,13$ \\
$-\cdots$ & $\begin{array}{l}\text { Percepción de alternativas, inversión en la organización Creencia de } \\
\text { Continuidad }\end{array}$ & $1,3,4,5,16,17$ \\
\hline
\end{tabular}

Para medir la Motivación se aplicó la escala de Motivación en el Trabajo (R-MAWS), diseñada por Gagné, Forest, Gilbert, Aubé, Morin \& Malorni en el año 2010; y adaptada al español por (Gagné et. al., 2012). Esta escala cuenta con 19 ítems que se agrupan en cinco subescalas que miden los diferentes tipos de motivación presentes al realizar una actividad: desmotivación, regulación externa, regulación introyectada, regulación identificada y motivación intrínseca. El instrumento registra las respuestas mediante una escala tipo Likert que va del 1 al 7 , siendo 1 totalmente en desacuerdo y 7 totalmente de acuerdo. 


\begin{tabular}{l|l|c}
\hline \multicolumn{1}{c}{ Dimensión } & \multicolumn{1}{c}{ Indicadores } & \multicolumn{1}{c}{ Preguntas } \\
\hline \multicolumn{1}{c}{ Desmotivación } & Ausencia de estímulos en el trabajo & $2,15,16$ \\
$-\begin{array}{l}\text { Regulación } \\
\text { Externa }\end{array}$ & $\begin{array}{l}\text { Respuesta a una demanda externa 0 capacidad de recibir una } \\
\text { recompensa impuesta por la organización }\end{array}$ & $3,8,9,10,14,17$ \\
\hline $\begin{array}{l}\text { Regulación } \\
\text { Introyectada }\end{array}$ & $\begin{array}{l}\text { Evadir castigos, o el miedo a perder su imagen propia actuan- } \\
\text { do bajo la presión de una medida externa }\end{array}$ & $1,5,7,19$ \\
\hline $\begin{array}{l}\text { Regulación } \\
\text { Identificada }\end{array}$ & Identificación del trabajador con la organización & $4,11,18$ \\
$-\begin{array}{l}\text { Motivación } \\
\text { Intrínseca }\end{array}$ & Autoconfianza, sentido de pertenencia a la organización & $6,12,13$ \\
\hline
\end{tabular}

\section{Validez y Confiabilidad}

Los instrumentos de investigación (TCM y R-MAWS) fueron administrados en cinco de los programas especiales descentralizados de la Universidad
Nacional de Piura (Paita, Sechura, Talara, Huancabamba y Chulucanas) de manera individual a 168 docentes, grupo distribuido proporcionalmente al tamaño de cada uno de los estratos.

Tabla 5. Estadísticas de Fiabilidad del Instrumento R-MAWS (Motivación)

\begin{tabular}{ccc}
\hline \multirow{2}{*}{ Motivación } & $\mathrm{N}^{\circ}$ Elementos & Alfa de Cronbach \\
& 19 & 0,939 \\
\hline
\end{tabular}

Fuente: Prueba Piloto Aplicada.

Diseño: Propio. 
Tabla 6. Estadísticas de Fiabilidad de los componentes del Instrumento R-MAWS (Motivación)

\begin{tabular}{lcc}
\hline Componente & N $^{\circ}$ Elementos & Alfa de Cronbach \\
Desmotivación & 3 & 0,852 \\
Regulación Externa & 6 & 0,887 \\
Regulación Introyectada & 4 & 0,824 \\
Regulación Identificada & 3 & 0,909 \\
Motivación Intrínseca & 19 & 0,883 \\
\hline
\end{tabular}

Fuente: Prueba Piloto Aplicada.

Diseño: Propio.

En las tablas se presentan los resultados que arroja el software estadístico Statgraphics Centurion respecto a una prueba piloto aplicada a 15 profesores de los programas especiales descentralizados de la Universidad Nacional de Piura.

Para la confiabilidad del instrumento, se ha calculado el coeficiente Alfa de Cronbach en los distintos constructos del cuestionario R-MAWS (escala de Motivación en el Trabajo) arrojando un coeficiente global de 0,939, lo que evidencia una alta confiabilidad del ins- trumento aplicado; se encontró 0,852 para desmotivación, 0,887 para regulación externa, 0,824 para regulación introyectada, 0,909 para regulación identificada y 0,883 para motivación intrínseca. Resultados que coinciden con los obtenidos originalmente por Gagné, Forest, Gilbert, Aubé, Morin \& Malorni en el año 2010; y adaptada al español por (Gagné et. al., 2012) en los que se asegura un coeficiente Alfa de Cronbach superior al 0,70. Garantizando la fiabilidad de la consistencia interna del cuestionario en los diversos constructos. 
Tabla 7. Estadísticas de Fiabilidad del Instrumento TCM

(Compromiso Organizacional)

\begin{tabular}{ccc}
\hline & $N^{\circ}$ Elementos & Alfa de Cronbach \\
Compromiso Organizacional & 18 & 0,932 \\
\hline
\end{tabular}

Fuente: Prueba Piloto Aplicada.

Diseño: Propio.

Tabla 8. Estadísticas de Fiabilidad de los componentes del Instrumento TCM (Compromiso Organizacional)

\begin{tabular}{lcc}
\hline Componente & N $^{\circ}$ Elementos & Alfa de Cronbach \\
Afectiva & 6 & 0,877 \\
Normativa & 6 & 0,919 \\
Continuidad & 6 & 0,813 \\
\hline
\end{tabular}

Fuente: Prueba Piloto Aplicada.

Diseño: Propio.

Las tablas anteriores dan cuenta de los resultados que arroja el software estadístico Statgraphics Centurion de la prueba piloto aplicada a 15 profesores de los programas especiales descentralizados de la Universidad Nacional de Piura; para la confiabilidad del instrumento, se ha calculado el coeficiente alfa de Cronbach en los distintos constructos del cuestionario TCM (Modelo de Tres Componentes del Compromiso) arro- jando un coeficiente global de 0,932, lo que evidencia una alta confiabilidad del instrumento aplicado; se encontró 0,877 para afectiva, 0,919 para normativa y 0,813 para continuidad. Resultados que coinciden con los obtenidos originariamente por Meyer y Allen (1997) en los que se determina un coeficiente Alfa de Cronbach de 0,82. Garantizando la fiabilidad de la consistencia interna del cuestionario en los diversos constructos. 


\section{RESULTADOS}

Tabla 9. Niveles de Motivación

\begin{tabular}{lcccccccc}
\hline & \multicolumn{2}{c}{ Baja } & \multicolumn{2}{c}{ Media } & \multicolumn{2}{c}{ Alta } & \multicolumn{2}{c}{ Total } \\
& $\mathrm{fi}$ & $\%$ & $\mathrm{fi}$ & $\%$ & $\mathrm{fi}$ & $\%$ & $\mathrm{fi}$ & $\%$ \\
\hline Desmotivación & 31 & 18,5 & 12 & 7,1 & 125 & 74,4 & 168 & 100,0 \\
\hline Regulación Externa & 39 & 23,2 & 96 & 57,1 & 33 & 19,6 & 168 & 100,0 \\
\hline Regulación Introyectada & 1 & 0,6 & 19 & 11,3 & 148 & 88,1 & 168 & 100,0 \\
\hline Regulación Identificada & 2 & 1,2 & 30 & 17,9 & 136 & 81,0 & 168 & 100,0 \\
\hline Motivación Intrínseca & 5 & 3,0 & 33 & 19,6 & 130 & 77,4 & 168 & 100,0 \\
\hline
\end{tabular}

Fuente: Cuestionario Aplicado a los docentes de los programas especiales descentralizados de la Universidad Nacional de Piura.

Diseño: Propio.

\section{Gráfico 1. Niveles de Motivación}

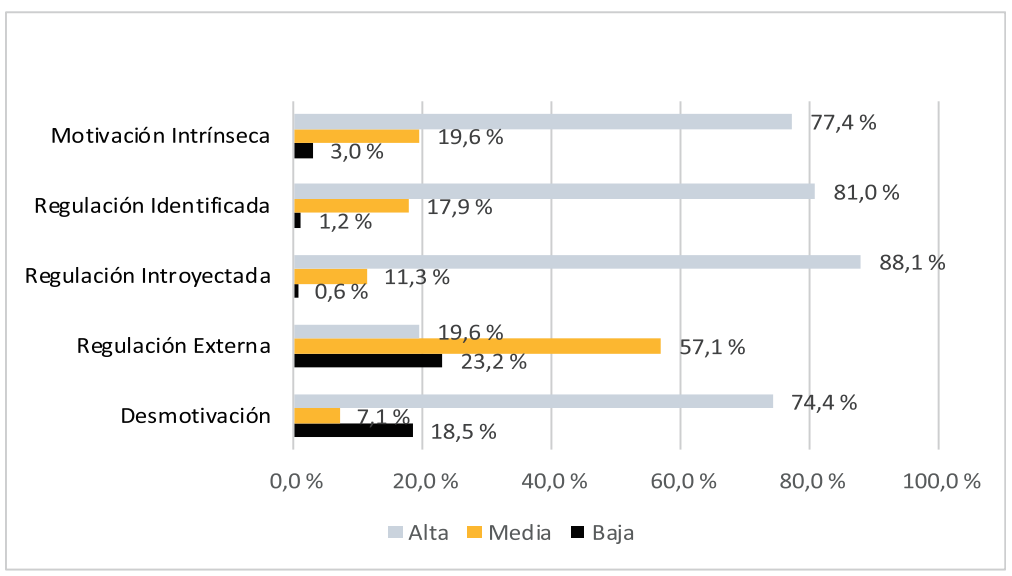

Fuente: Excel V. 16.0.

Diseño: Propio.

La tabla y gráfica anteriores descentralizados de la Universidad Namuestran el nivel de motivación de los cional de Piura; los resultados dejan claro profesores de los programas especiales que este es bastante favorable principal- 
mente en las dimensiones motivación intrínseca, regulación identificada y regulación introyectada donde se presentan niveles sumamente altos (llegando a superar el 80,0\%). En lo que corresponde a la regulación externa existe una valo- ración media de esta dimensión. Asimismo, hay evidencia de ausencia en los estímulos (desmotivación) al momento de realizar sus actividades, indicador preocupante que alcanza un 74,4 \%.

Tabla 10. Niveles de Compromiso Organizacional

\begin{tabular}{lcccccccc}
\hline & \multicolumn{2}{c}{ Baja } & \multicolumn{2}{c}{ Media } & \multicolumn{2}{c}{ Alta } & \multicolumn{2}{c}{ Total } \\
& $\mathrm{Fi}$ & $\%$ & $\mathrm{Fi}$ & $\%$ & $\mathrm{fi}$ & $\%$ & fi & $\%$ \\
\hline Afectiva & 10 & 6,0 & 122 & 72,6 & 36 & 21,4 & 168 & 100,0 \\
\hline Normativa & 16 & 9,5 & 65 & 38,7 & 87 & 51,8 & 168 & 100,0 \\
\hline Continuidad & 22 & 13,1 & 125 & 74,4 & 21 & 12,5 & 168 & 100,0 \\
\hline
\end{tabular}

Fuente: Cuestionario Aplicado a los docentes de los programas especiales descentralizados de la Universidad Nacional de Piura.

Diseño: Propio.

\section{Gráfico 2. Niveles de Compromiso Organizacional}

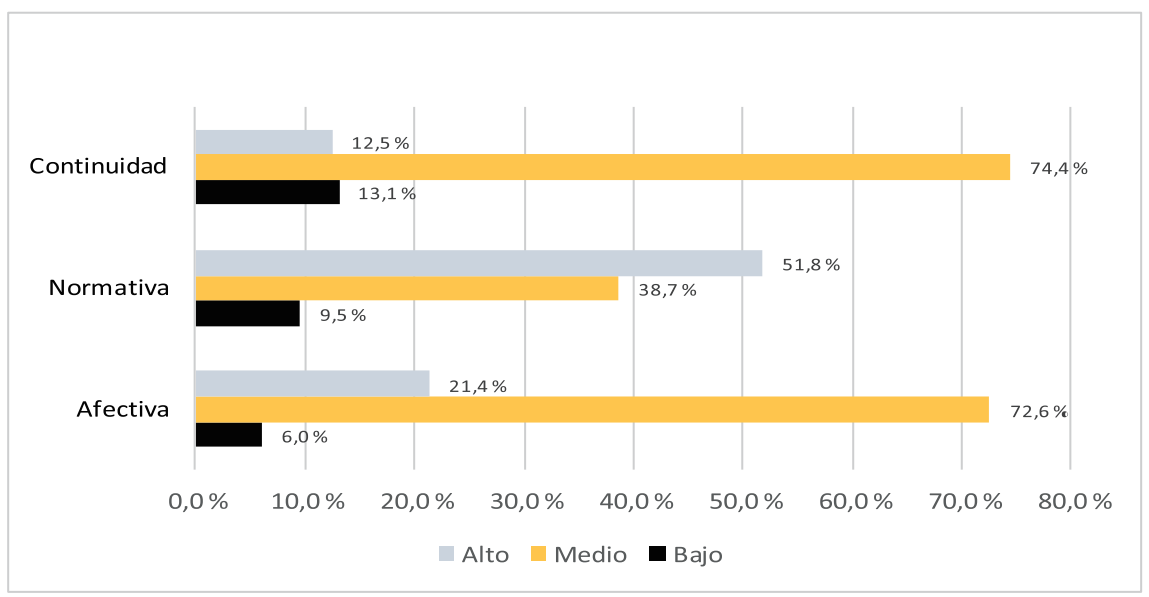

Fuente: Excel V. 16.0

Diseño: Propio 
La tabla y gráfica anteriores muestran el nivel de compromiso organizacional que en opinión de los encuestados es el que prevalece durante el desarrollo de sus actividades. Los resultados conseguidos son bastante favorables, principalmente, en la dimensión de normativa que alcanza valores relativamente altos representados por el 51,8 \% de nuestra muestra representativa. La dimensión de continuidad alcanza valores medios de compromiso organizacional con un $74,4 \%$. Asimismo, la dimensión afectiva alcanza valores medios representados por el $72,6 \%$.

Tabla 11. Relación entre Motivación y Compromiso Organizacional

\begin{tabular}{|c|c|c|c|}
\hline & & & Compromiso Organizacional \\
\hline \multirow{2}{*}{ Rho de Spearman } & \multirow{2}{*}{ Motivación } & Coeficiente de correlación &, 638 \\
\hline & & Significancia (bilateral) &, 000 \\
\hline
\end{tabular}

Fuente: Cuestionario aplicado a los docentes de los programas especiales descentralizados de la Universidad Nacional de Piura.

Diseño: Propio.

\section{Gráfico 3. Correlación entre Motivación y Compromiso Organizacional}

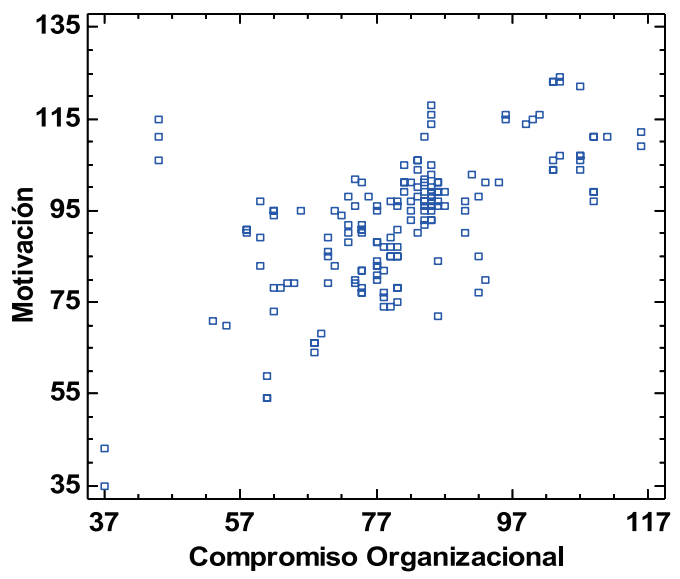

Fuente: Statgraphics Centurion XVI.

Diseño: Propio. 
La correlación de Spearman entre motivación y compromiso organizacional es 0,638; lo que indica que existe una relación positiva entre las variables.

El análisis de correlación da cuenta que la motivación muestra una relación significativa (Sig. $<0,05)$ con el compromiso organizacional de profeso- res de los programas especiales descentralizados de la Universidad Nacional de Piura. Estos resultados conducen a aceptar la hipótesis general de investigación la cual sugiere que entre la motivación y el compromiso organizacional existe una influencia significativa.

\section{DISCUSIÓN}

Con relación al primer objetivo, el cual es determinar el grado de motivación de los docentes de los Programas Especiales Descentralizados UNP, en el año 2017; los resultados dejan claro que este es bastante favorable principalmente en las dimensiones motivación intrínseca, regulación identificada y regulación introyectada donde se presentan niveles sumamente altos (llegando a superar el $80,0 \%$ ). Asimismo, hay evidencia de ausencia en los estímulos (desmotivación) al momento de realizar sus actividades, indicador preocupante que alcanza un $74,4 \%$; lo que presenta similitudes con lo encontrado por Pedroza (2007), en su tesis, "Compromiso organizacional, motivación de logro y satisfacción laboral en los trabajadores públicos en Caracas", donde se observó que la variable compromiso organizacional y motivación al logro se correlacionan de forma significativa ( $p<, 05$ ), observándose una corre- lación entre ambas escalas de $r=, 142$. De igual forma se evidencia que a mayor asertividad en la motivación al logro, mayor es el compromiso afectivo con la empresa $(r=, 143)$ y la permanencia en la misma ( $r=, 248)$; por otra parte, mientras exista una mayor motivación de logro en excelencia menor es el compromiso de permanencia $(r=-, 179)$, y todo ello fundamentado en las teorías de Santrock (2002), quien describe a la motivación como una sumatoria de razones por las cuales el individuo se comporta de determinada forma y de López (2009), quien afirma que la motivación explica la intensidad y persistencia de un tipo de comportamiento orientado al cumplimiento de objetivos determinados.

En relación al segundo objetivo el cual es determinar el grado de compromiso organizacional de los docentes de los Programas Especiales Descentralizados UNP, en el año 2017; en esta inves- 
tigación tenemos como resultado que el nivel de compromiso organizacional es el que prevalece durante el desarrollo de sus actividades; resultados similares a lo obtenido por Crespo (2016), quien investigó el clima y compromiso organizacional donde para evaluar el compromiso organizacional, usó el Cuestionario de Compromiso Organizacional de Meyer y Allen. Y gracias a la aplicación de ello se evidencia un predominante nivel alto del $73 \%$ en relación al compromiso organizacional. Asimismo no se pudo establecer similitudes con Bernal (2015), quien investigó el compromiso de logro en empleados de una corporación y en los resultados muestran que existen diferencias entre el compromiso organizacional por sexo, siendo las mujeres quienes presentan un compromiso mayor, lo que las motiva a cumplir con las metas propuestas.

En relación al siguiente objetivo el cual es establecer la relación existente entre la desmotivación y el compromiso organizacional de los docentes de los Programas Especiales Descentralizados PROEDUNP's de la Universidad Nacional de Piura, en el año 2017, se encontró un Coeficiente de correlacion de Sperman de $-0,341$; lo que indica que existe una relación negativa entre estas variables. Según lo señalado en el marco teórico esto se refiere a la ausencia de estímulos, no encontrándose similitudes en los es- tudios previos nos conlleva a establecer que esta investigación es una pionera en el estudio y evaluación de este aspecto.

En relación al objetivo denominado establecer la relación existente entre la regulación externa y el compromiso organizacional de los docentes de los Programas Especiales Descentralizados PROEDUNP's de la Universidad Nacional de Piura, en el año 2017, se tiene un Coeficiente de correlacion de Sperman de 0,409 , lo que indica que existe una relación positiva entre estas variables; existe una valoración media de esta dimensión, ello debido a que al ser en su gran mayoría docentes contratados, no pueden recibir ningún tipo de bonificación, lo antes expuesto se clarifica con los conceptos mostrados en el marco teórico de esta investigación en el cual se define como respuesta a una demanda externa o capacidad de recibir una recompensa impuesta por la organización.

El siguiente objetivo definido como establecer la relación existente entre la regulación introyectada y el compromiso organizacional de los docentes de los Programas Especiales Descentralizados - PROEDUNP's de la Universidad Nacional de Piura, en el año 2017, donde se obtuvo un Coeficiente de correlacion de Sperman de 0,234, lo que indica que existe una relación positiva entre estas variables; se presentan niveles sumamente altos, ello debido a que todos los 
docentes se rigen por lo establecido en el reglamento académico, reglamento general, el estatuto y la ley universitaria. Ello tiene coherencia con lo presentado en el marco teórico de la investigación lo cual implica evadir castigos, o el miedo a perder su imagen propia actuando bajo la presión de una medida externa.

El siguiente objetivo era establecer la relación existente entre la regulación identificada y el compromiso organizacional de los docentes de los Programas Especiales Descentralizados PROEDUNP's de la Universidad Nacional de Piura, en el año 2017. Se obtuvo un Coeficiente de correlacion de Sperman de 0,383; lo que indica que existe una relación positiva entre estas variables, relacionándola de una forma óptima con el marco teórico donde se define como la identificación del trabajador con la organización.

En el objetivo específico siguiente fue establecer la relación existente entre la motivación intrínseca y el compromiso organizacional de los docentes de los Programas Especiales Descentralizados PROEDUNP's de la Universidad Nacional de Piura, en el año 2017; se obtuvo un Coeficiente de correlación de Sperman de 0,298 , lo que indica que existe una relación, los docentes tienen los niveles de profesionalismo suficiente para generar confianza en ellos mismos y sentirse seguros al desarrollar sus actividades, los docentes sienten un grado alto de pertenencia en la institución debido al prestigio que esta tiene a nivel regional y nacional, ello tiene relación con lo expresado por Deci \& Ryan (2000), quienes al remitirse a la motivación intrínseca enfatizan el nexo entre la persona y la tarea, el cual puede ser calificado, según algunos autores, como interés y según otros, como compromiso.

Finalmente, respecto al objetivo principal: establecer la relación entre la motivación y el compromiso organizacional de los docentes de los programas especiales descentralizados de la Universidad Nacional De Piura, en el año 2017, se establece que la relación existente entre la motivación y el compromiso organizacional de dichos docentes, según el coeficiente de correlación de Spearman es de 0,638; lo que indica que existe una relación positiva entre las variables; este resultado es similar a los obtenidos por Gonzales (2015) cuando investigó el compromiso laboral y su relación con el reconocimiento, y que indica que existe una relación directa entre ambas variables; asimismo se determinó que el $63 \%$ presentaba un adecuado compromiso laboral y el $16 \%$ presentaba bajos niveles de compromiso; y con Marquina (2013) quien investigó la satisfacción laboral y el compromiso organizacional en personal perteneciente a una universidad, los resultados evidencian una rela- 
ción altamente significativa entre ambas variables; se evidencia un compromiso elevado del $91 \%$, asimismo el $66 \%$ se siente comprometido para participar en las diversas actividades, el $84,2 \%$ se identifica con la universidad, el 81,1\% presenta el sentido de pertenencia y el $66,1 \%$ es leal con la organización.

\section{CONCLUSIONES}

Con respecto a la motivación que sienten los Docentes de los programas especiales descentralizados de la Universidad Nacional de Piura al momento de desarrollar sus actividades se han obtenido valoraciones positivas, todo ello teniendo como punto más importante la identificación con la institución y teniendo como base el profesionalismo de los mismos, sin embargo la mayor parte de los docentes no están de acuerdo con la compensación en términos económicos por el trabajo desarrollado, ello debido a que en la institución el pago no es oportuno.

La motivación se encuentra en niveles favorables principalmente en las siguientes dimensiones:

- Motivación intrínseca donde los docentes tienen los niveles de profesionalismo suficiente para generar confianza en ellos mismos y sentirse seguros al desarrollar sus actividades, los docentes sienten un grado alto de pertenencia en la institución debido al prestigio que esta tiene a nivel regional y nacional.
- Regulación identificada en lo que respecta al comportamiento de los docentes y al cumplimiento de sus actividades, ello denota un grado alto de identificación del trabajador con la organización.

- Regulación introyectada donde se presentan niveles sumamente altos, ello debido a que todos los docentes se rigen por lo establecido en el reglamento académico, reglamento general, el estatuto y la ley universitaria.

En lo que corresponde a la regulación externa existe una valoración media de esta dimensión, ello debido a que al ser en su gran mayoría docentes contratados, no pueden recibir ningún tipo de bonificación, lo que también genera ausencia en los estímulos (desmotivación) al momento de realizar sus actividades, ya que la motivación solo es económica mas no de formación académica - profesional.

Teniendo así que los factores motivacionales de los docentes de los Programas Especiales Descentralizados 
UNP, en el año 2017, han sido factores intrínsecos en su mayoría en relación a la oportunidad de ser miembros de una institución de prestigio, la aplicación de lo normado también es una variable de motivación encontrada, así como la confianza en sí mismos.

Con respecto al compromiso organizacional y a la convivencia dentro del entorno de trabajo, circunstancias de cercanía e identificación con la institución, así como de la predisposición de desarrollar sus actividades como hasta ahora, el $100 \%$ de los encuestados demuestran gratitud para con la institución ya que reconocen el respaldo obtenido frente a alguna circunstancia vivida, debido al prestigio de esta institución y que genera con ello elevar su nivel profesional; asimismo debo señalar que debido a que gran cantidad de docentes son contratados se establece que la continuidad en el mediano y largo plazo es nula.

Asimismo, se debe señalar que respecto al nivel de compromiso organizacional, los resultados conseguidos son bastante favorables principalmente en la dimensión de normativa donde alcanza valores altos en lo que respecta a lealtad, sentido de organización y sentido de obligación, todo ello basado en el esfuerzo que los docentes hacen en la planeación, organización, dirección y evaluación de sus clases, ya que estos programas son lejanos y en su mayoría los docentes se trasladan desde 45 minutos hasta la posibilidad de pasar días en la localidad asignada.

Además, debo señalar que en lo que respecta a las posibles alternativas de continuidad y al temor de dejar la institución, los valores encontrados son medios. Y finalmente, en este apartado debo establecer que el deseo de pertenecer a la organización y de seguir en ella también presenta niveles favorables para la institución.

Como conclusión final se establece que la relación existente entre la motivación y el compromiso organizacional de los docentes de los Programas Especiales Descentralizados - PROEDUNP's de la Universidad Nacional de Piura, en el año 2017, según el coeficiente de correlación de Spearman, es de 0,638; lo que indica que existe una relación positiva entre las variables. 


\section{REFERENCIAS BIBLIOGRÁFICAS}

Allen, N.J. y Meyer, J.P. (1990) the measurement of antecedents of affective, continuance and normative commitment to the organization, Journal of Occupational Psychology, 63 (1) 1-18. doi: 10.1111/ j.2044-8325.1990.tb00506.x

Allen, N. J., \& Meyer, J.P. (1996). Affective, continuance, and normative commitment to the organization: An examination of construct validity. Journal of Vocational Behavior, 49, 252-276. doi: 10.1006/ jvbe.1996.0043,

Deci, E. L., \& Ryan, R. M. (2000). The "what" and "why" of goal pursuits: Human needs and the self-determination of behavior. Psychological Inquiry, 11, 227-268.

Gagné, M., \& Deci, E. L. (2012). Self-determination theory as a new framework for understanding organizational behavior. Journal of Organizational Behavior, 26, 331-362 doi: 10.1002/job.322

Meyer, J.P.y Allen, N.J. (1997) A three-component conceptualization of organizational commitment. Human Resource Management Review, 1, 61-89 doi: 10.1016/10534822(91)90011-Z.

Meyer, J.P. \& Gagné, M. (2008) Employee Engagement from a Self-determination Theory Perspective. Industrial and Organizational Psychology, 1,60-62 doi: 1754- 9426/08.

Zamora, P. G. (2009) Compromisos organizacionales de los profesores chilenos y su relación con la intención de permanecer en sus escuelas. Revista Latinoamericana de Psicología, 41 (3) 445-460. 